\title{
Synthesis of Dextran/Methoxy Poly(ethylene glycol) Block Copolymer
}

\author{
Young-Il Jeong, ${ }^{1}$ Dong-Gon Kim, ${ }^{2}$ and Dae-Hwan Kang ${ }^{1}$ \\ ${ }^{1}$ National Research and Development Center for Hepatobiliary Disease, Pusan National University Yangsan Hospital, \\ Beomeo-ri, Mulgeum-eup, Yangsan, Gyeongnam 626-770, Republic of Korea \\ ${ }^{2}$ Utah-Inha DDS \& Advanced Therapeutics Research Center, Incheon 406-840, Republic of Korea
}

Correspondence should be addressed to Dae-Hwan Kang; sulsulpul@naver.com

Received 26 April 2013; Revised 8 July 2013; Accepted 18 August 2013

Academic Editor: Mehdi Rajabi

Copyright (C) 2013 Young-Il Jeong et al. This is an open access article distributed under the Creative Commons Attribution License, which permits unrestricted use, distribution, and reproduction in any medium, provided the original work is properly cited.

\begin{abstract}
We synthesized a block copolymer composed of dextran and methoxy poly(ethylene glycol) (mPEG). To accomplish this, the end group of dextran was modified by reductive amination. The aminated dextran (Dextran- $\mathrm{NH}_{2}$ ) showed the intrinsic peaks of both dextran at $3 \sim 5.5 \mathrm{ppm}$ and hexamethylene diamine at $1 \sim 2.6 \mathrm{ppm}$ at ${ }^{1} \mathrm{H}$ nuclear magnetic resonance (NMR) spectrum. The amino end group of dextran was conjugated with $\mathrm{mPEG}$ to make the block copolymer consisting of dextran/mPEG (abbreviated as DexPEG). The synthesized aminated dextran and DexPEG were characterized using ${ }^{1} \mathrm{H}$ NMR and gel permeation chromatography (GPC). The molecular weight and conjugation yield were estimated by comparing the intensity ratio of the proton peaks of the glucose molecule (4.9 ppm and 3.3 4.0 ppm) to that of the ethylene group of mPEG (3.7 ppm). Abundant hydroxyl group in the dextran chain can be used as a source of bioactive agent conjugation.
\end{abstract}

\section{Introduction}

Dextran is a colloidal and hydrophilic macromolecule. Since dextran has immunoneutrality, it has been extensively used in the biomedical field and drug delivery applications [1-7]. Especially, dextran is known to be one of the most useful mediators for the conjugation of bioactive agents in targeted drug delivery systems $[4,6]$. Since dextran is degradable in the colon by the colonic enzyme, it is considered to be one of the most important materials for colonic drug targeting [4]. Sugahara et al. [5] reported that carboxymethyl dextran conjugated anticancer agents significantly suppressed tumor growth in the animal tumor xenograft model. Furthermore, dextran is known to be taken up in the intestinal tract in a specific manner [7]. Pérez et al. [8] reported that catalaseconjugated dextran increases the enzymatic activity and bioavailability.

The modification of dextran itself was also reported by numerous researchers to enhance its value as a biomaterial [9-13]. Van Dijk-Wolthuis et al. [9] reported the use of glycidyl methacrylate derivatized dextran for biomedical applications. Hydrogel or nanoparticles of dextranPEG macromer were reported to be useful vehicles for protein or anticancer drugs [10, 11]. Rutot et al. [12] reported an amphiphilic copolymer composed of poly $(\varepsilon$ caprolactone) and dextran. Furthermore, we previously reported that dextran-block-poly(DL-lactide-co-glycolide) (PLGA) copolymer has amphiphilic properties and it can form core-shell type nanoparticles for anticancer drug delivery [13].

In this study, we synthesized and characterized an mPEG/dextran (DexPEG) block copolymer. Even though Hernandez et al. [14, 15] previously reported an mPEG/dextran block copolymer, no detailed characterization of it was performed. Proton NMR and GPC were used for the characterization of the DexPEG block copolymer.

\section{Materials and Methods}

2.1. Materials. Dextran from Leuconostoc mesenteroides (molecular weight: 18,000 g/mol) was purchased from Sigma Chem. Co. (St. Louis, USA). Sodium cyanoborohydride was purchased from Fluka. Co. USA. Hexamethylene diamine (HMDA) was purchased from Aldrich Chemical Co. USA. Methoxy poly(ethylene glycol) N-hydroxy succinimide 
$(\mathrm{mPEG}-\mathrm{NHS}$, M.W. $=2,000 \mathrm{~g} / \mathrm{mol})$ was purchased from SunBio Co. Korea. Dialysis membrane with a molecular weight cutoff (MWCO) of $8,000 \mathrm{~g} / \mathrm{mol}$ was purchased from Spectra/Pro Membranes. Dichloromethane (DCM) and dimethyl sulfoxide (DMSO) were of HPLC grade and used without further purification.

\subsection{Synthesis of mPEG/Dextran (DexPEG) Block Copoly-} mer. Aminated dextran: $180 \mathrm{mg}$ of dextran was dissolved in DMSO. To this solution, sodium cyanoborohydride and HDMA were added. This mixture was stirred for $24 \mathrm{~h}$ at room temperature. After that, 10 equivalents of HMDA was added to the above dextran solution and then the mixture was further stirred for $24 \mathrm{~h}$ at room temperature. This solution was dialyzed against deionized water using dialysis membrane (MWCO: $8,000 \mathrm{~g} / \mathrm{mol}$ ) for 3 days and lyophilized for 3 days. Dextran-HMDA conjugates were used as an aminated dextran, and HMDA at the end of dextran chain was confirmed by $1 \mathrm{H}$ NMR spectroscopy.

DexPEG block copolymer: aminated dextran was dissolved in DMSO and 1.5 equivalents of mPEG-NHS was added to the above dextran solution. This mixture was further stirred for $24 \mathrm{~h}$ at room temperature. After that, the resulting solution was introduced into a dialysis tube (MWCO: $8,000 \mathrm{~g} / \mathrm{mol}$ ) and dialyzed against a large amount of deionized water for 2 days. Water was exchanged every $2 \mathrm{~h}$ to remove the organic solvent and unreacted $\mathrm{mPEG}$ NHS. To remove remaining unreacted $\mathrm{mPEG}$, resulting solid was precipitated into chloroform and then filtered. This purification step was repeated three times and dried in vacuum for 3 days. A white solid product was obtained by the lyophilization of this solution for 3 days.

2.3. ${ }^{1} \mathrm{H}$ Nuclear Magnetic Resonance Spectroscopy (NMR) Measurement. The ${ }^{1} \mathrm{H}$ NMR spectra of the copolymers were measured in DMSO or $\mathrm{D}_{2} \mathrm{O}$ using a $400 \mathrm{MHz}$ NMR spectrometer (Varian $400 \mathrm{MHz}$ NMR).

2.4. Gel Permeation Chromatography (GPC). The absolute molecular weight and MW distribution, represented by the polydispersity index (PD), of the DexPEG block copolymer were measured using a GPC equipped with a multiangle laser light scattering detector (GPC-MALLS, 18 angle detector, Wyatt, USA) and column for water soluble polymer (OHpak SB-803HQ, Wyatt, USA). The samples were dissolved in $0.5 \mathrm{M}$ ammonium acetate buffer $(\mathrm{pH} 5.5)$ at more than 5 different concentrations ranging from 0 to $1.0 \mathrm{mg} / \mathrm{mL}$, and the change in the reflective index $(\mathrm{dn} / \mathrm{dc})$ was measured by means of a Pot-LAB reflectometer (Wyatt, USA). Then, the absolute MW and MW distribution of the DexPEG copolymer were obtained from the GPC chromatogram with the light scattering data (Debye plot regressions). The mobile phase was $0.5 \mathrm{M}$ ammonium acetate buffer ( $\mathrm{pH}$ 5.5), and the flow rate was $0.5 \mathrm{~mL} / \mathrm{min}$. The injection volume was $0.2 \mathrm{~mL}$ $(10 \mathrm{mg} / \mathrm{mL})$. The standard used for the determination of the MW of the copolymer was poly(ethylene glycol) (PEG).

\section{Results and Discussion}

Block copolymers have been extensively used for drug delivery applications due to their unique structure. Normally, block copolymers composed of hydrophilic and hydrophobic domains can form polymeric micelles in an aqueous environment; that is, the hydrophobic block can form the inner-core of the polymeric micelle, while the hydrophilic block forms its outershell [16]. Block copolymers composed of dextran and other polymers have also been reported. Pérez et al. [8] reported the improved pharmacokinetic properties of catalase-conjugated dextran. The catalase-conjugated dextran has similar architecture to block copolymer. Especially, the plasma half-life of their dextran-catalase conjugates was significantly increased. Bosker et al. [17] reported the synthesis of polystyrene-polysaccharide block copolymers. They also synthesized a dextran-polystyrene block copolymer, in which dextran is regarded as the hydrophilic domain and polystyrene as the hydrophobic domain.

We synthesized a block copolymer composed of dextran/mPEG and characterized it with proton NMR and GPC. Since both dextran and mPEG have a hydrophilic nature, the DexPEG block copolymer must be freely soluble in aqueous solution. However, a large amount of hydroxyl functional groups exists in the dextran domain and this functional group can provide a useful source of chemical conjugation or ion complexation with bioactive agents $[4-6,14,15]$. For example, functional moieties such as carboxyl group or amine group can be introduced into a hydroxyl group of dextran and these functional group can be used as a source of conjugation with bioactive agents $[14,15]$. Hernandez et al. synthesized block copolymers composed of carboxymethyl dextran (CM dextran) and mPEG [14]. They reported the $\mathrm{pH}$-responsive assembly of a double hydrophilic block copolymer of CM dextran and PEG. Furthermore, they reported that block copolymer composed of CM dextran and mPEG is able to form polyionic micelles with hydrophilic cationic drug and to use as a drug delivery vehicle [15]. Ichinose et al. reported that CM dextran-cisplatin conjugates showed a significantly higher antitumor activity than cisplatin alone [6]. Therefore, the dextran domain can act as a drug-incorporation site and has biodegradable properties in the human body. For example, the conjugation of hydrophobic drugs to the hydroxyl group of dextran may endow it with a hydrophobic nature and the resulting dextran-drug conjugate domain may act as a hydrophobic domain. Kim et al. [18] previously reported the formation of a polymeric micelle between mPEG-grafted chitosan and all-trans retinoic acid. Since they used watersoluble chitosan and their mPEG-grafted chitosan is a fully water-soluble copolymer, the polymer itself does not form a polymer micelle; that is, polymer micelles were only formed by ion-complex formation between chitosan and all-trans retinoic acid.

To synthesize the block copolymer, the end group of dextran was modified by reductive amination using sodium cyanoborohydride and HMDA, as shown in Figure 1. Since dextran itself did not have active site for conjugation with $\mathrm{mPEG}$, the reductive end of dextran was modified to have amine group. Amine end group of dextran has several 
TABLE 1: Characterization of the DexPEG block copolymer.

\begin{tabular}{lccccc}
\hline & Mn by ${ }^{1} \mathrm{H}-\mathrm{NMR}$ & \multicolumn{2}{c}{ Molecular weight by GPC } & \multicolumn{2}{c}{ Conjugation yield (\%) } \\
& & $\mathrm{Mn}$ & $\mathrm{Mw}$ & Polydispersity & NMR $^{\mathrm{a}}$ \\
\hline Dextran & - & 14,390 & 16,950 & $1.177 \pm 0.054$ & - \\
Dextran-NH & 14,505 & 14,680 & 18,050 & $1.229 \pm 0.057$ & 99.2 \\
DexPEG & 16,086 & 16,060 & 20,270 & $1.262 \pm 0.104$ & 84.8 \\
\hline
\end{tabular}

Mn: number-average molecular weight; Mw: weight-average molecular weight.

${ }^{a}$ Conjugation yield (CY) was evaluated by comparison of sum of proton peak (proton 1 position of dextran) of dextran and sum of proton peaks of hexamethylene diamine or $\mathrm{mPEG}$.

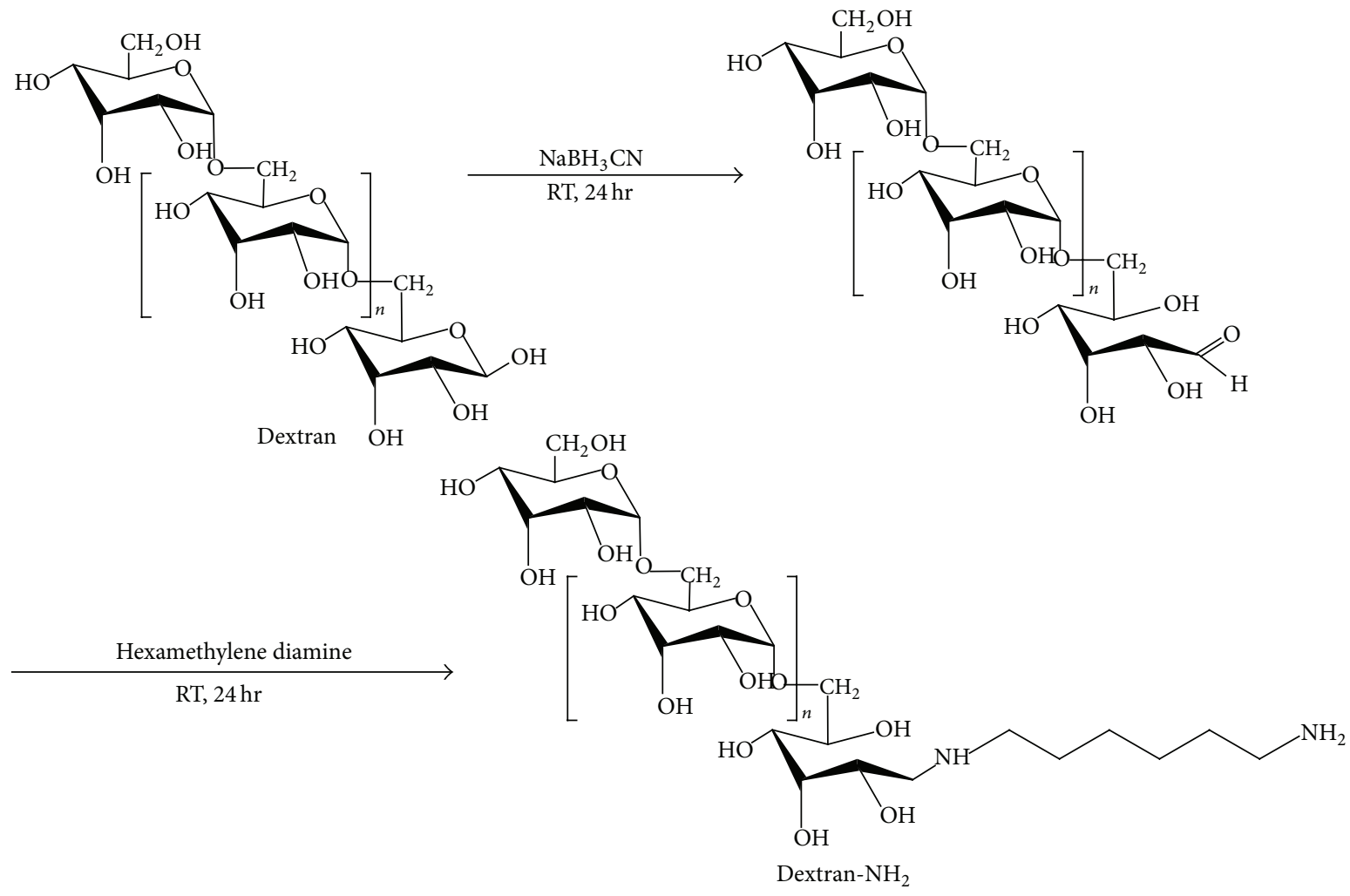

Figure 1: Synthesis scheme of dextran-hexamethylene diamine conjugate.

advantages. For example, the amine group of polymers can be easily conjugated with carboxylic acid by aid of carbodiimide chemistry and formed peptide bond. Furthermore, we already reported the successive synthesis of block copolymer composed of dextran and poly(DL-lactide-coglycolide) (PLGA) by using reductive amination of dextran [13]. To prevent dextran-dextran conjugation by reductive amination, an excess amount of HMDA was added and the aminated dextran was purified by a dialysis procedure. The aminated dextran was characterized using proton NMR as shown in Figure 2. As shown in Figure 2, dextran has intrinsic peaks at 3 5 ppm while HMDA has intrinsic peaks at 1.0 1.5. HMDA was attached to the end of dextran through reductive amination. The unreacted HMDA was removed by a dialysis method against distilled water. As shown in Figure 2, the aminated dextran (Dextran- $\mathrm{NH}_{2}$ ) showed the intrinsic peaks of both dextran at 3 5.5 ppm and HMDA at 1 2.6 ppm. Since almost all of the protons at the 1 position of dextran can be assumed to be similar before and after their conjugation with HMDA, the ratio of the peak intensity of the protons at the 1 position of dextran to that of HMDA was determined and used to estimate the M.W. and conjugation yield. The conjugation yield was greater than $99 \%$ at the end of dextran. As shown in Table 1, the average M.W. of the aminated dextran was slightly higher than that of dextran, even though the difference in their M.W.'s did not exactly reflect the M.W. of HMDA. These results clearly indicated that HMDA was successfully conjugated to the end of dextran.

To prepare the block copolymer, mPEG-NHS was attached to the amino end group of dextran, as shown in Figure 3. An excess amount of mPEG-NHS was reacted with the aminated dextran. The intrinsic peaks of dextran- $\mathrm{NH}_{2}$ at $1 \sim 5.5 \mathrm{ppm}$ and PEG-NHS at 1 4.5 ppm were defined, as shown in Figure 4 . The DexPEG block copolymer showed the intrinsic peaks of both $\mathrm{mPEG}$ and dextran. These results indicate that mPEG was successfully attached to the end 
(a)

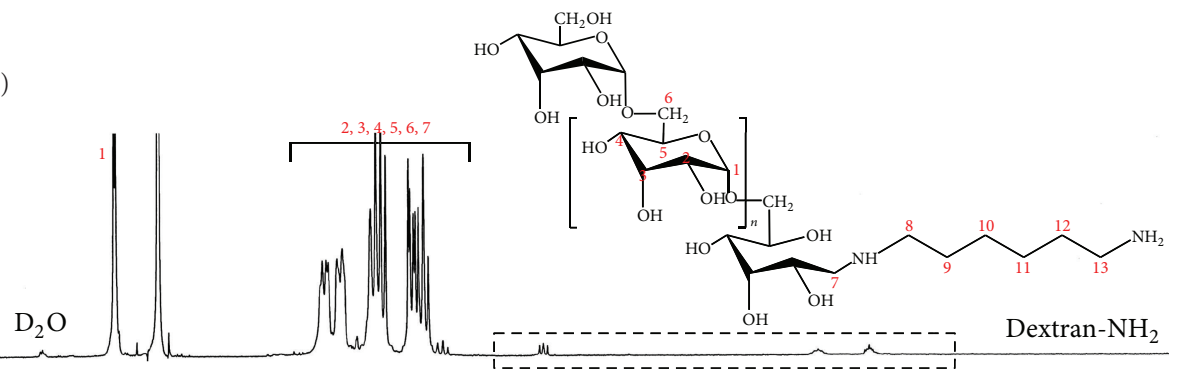

(b)
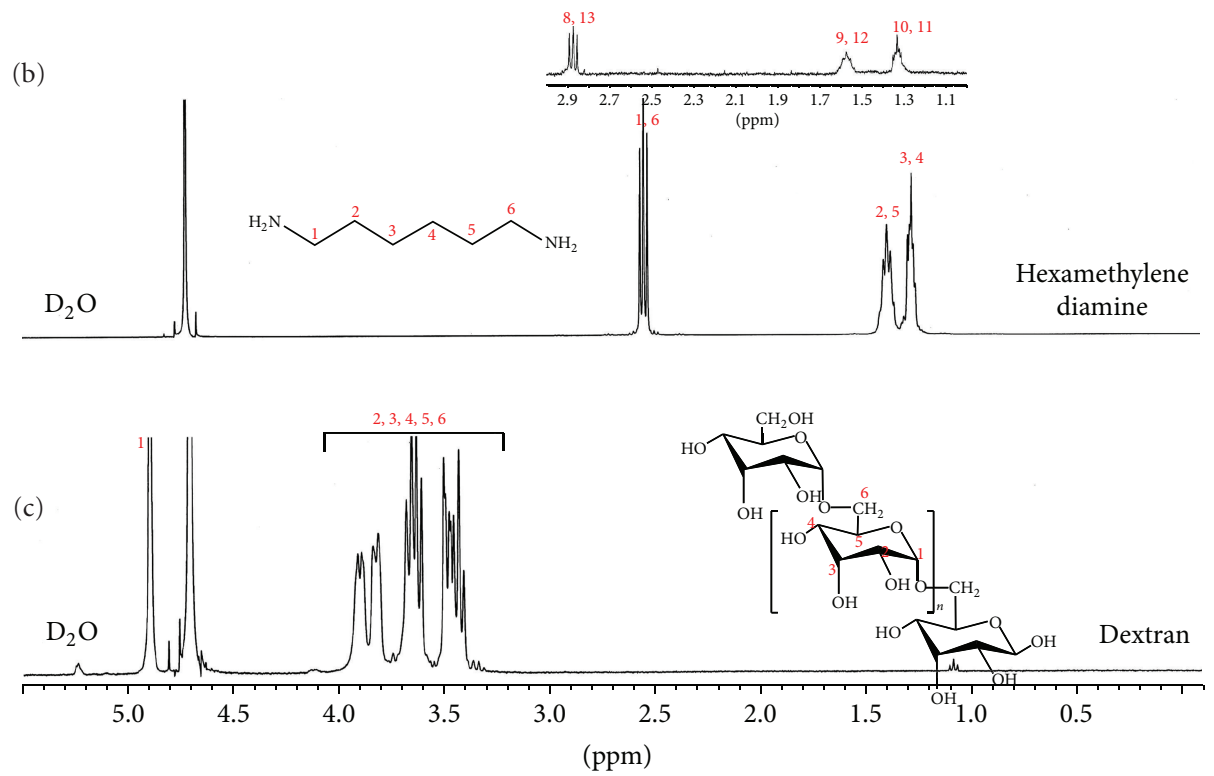

Figure 2: ${ }^{1} \mathrm{H}$ spectra of dextran (a), hexamethylene diamine (b), and dextran- $\mathrm{NH}_{2}$ (c) in $\mathrm{D}_{2} \mathrm{O}$.

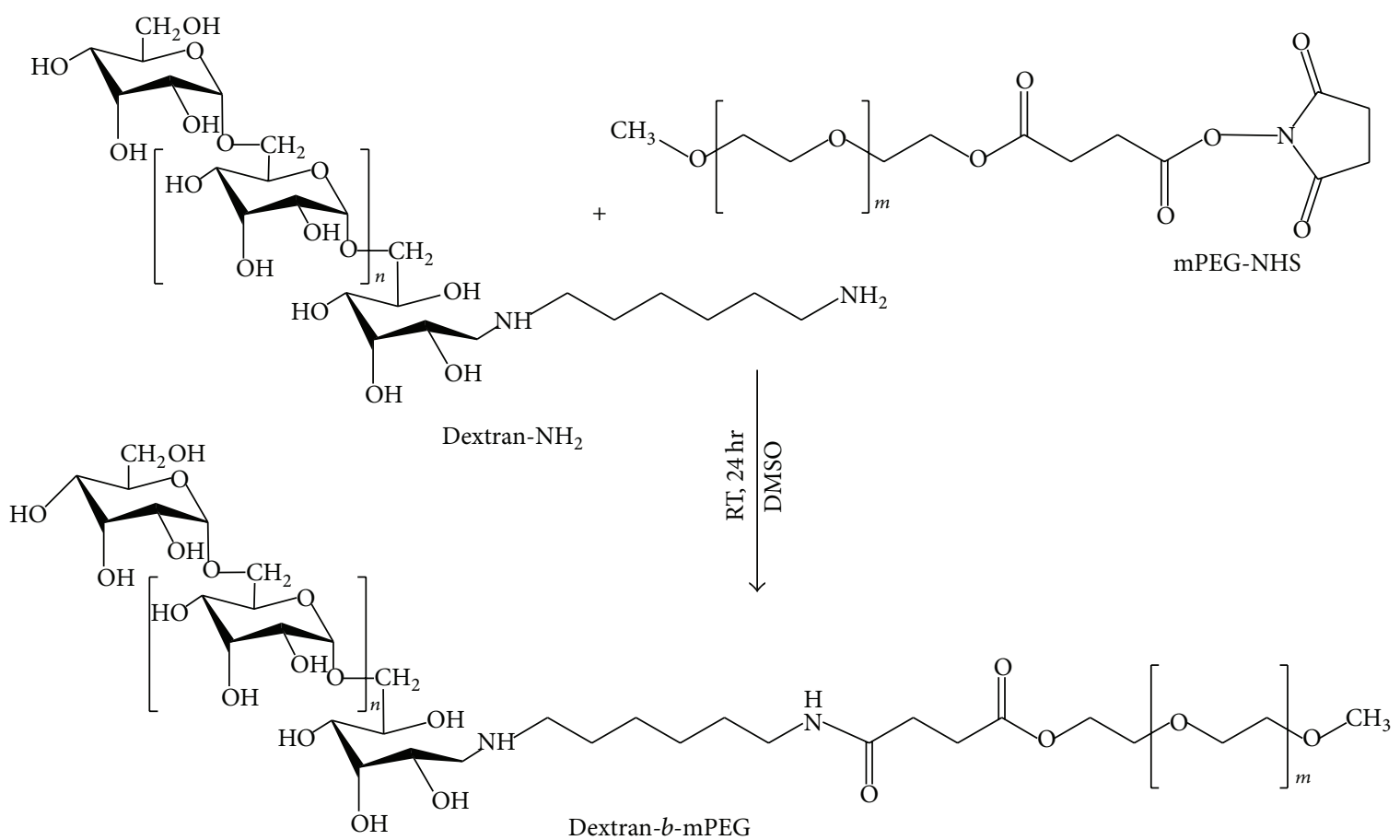

FIGURE 3: Synthesis scheme of DexPEG block copolymer. 
(a)

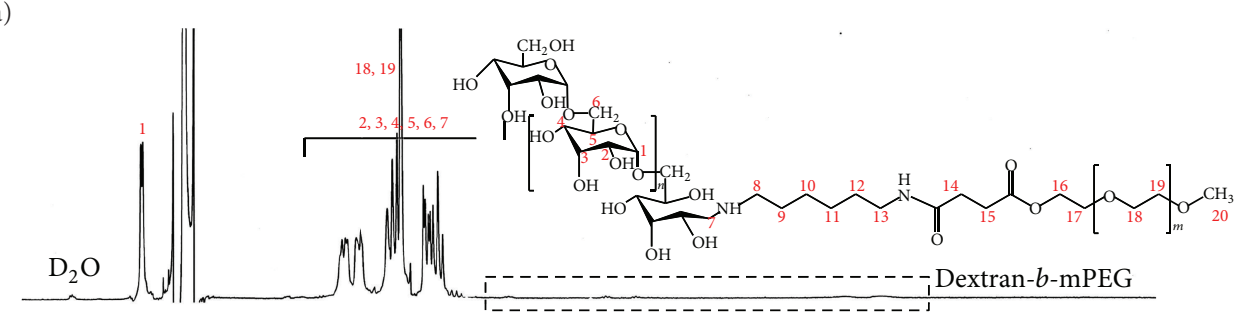

(b)

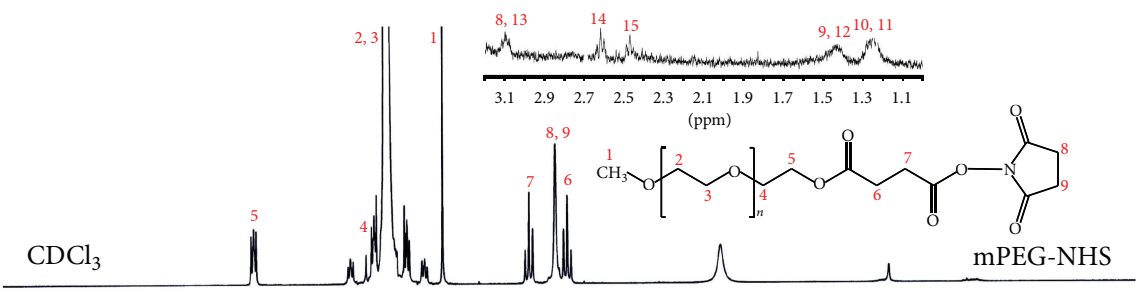

(c)

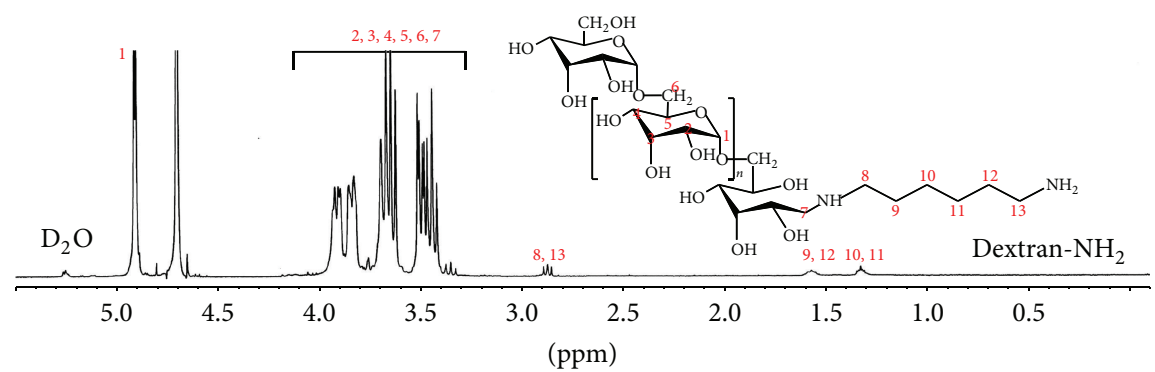

FIGURE 4: ${ }^{1} \mathrm{H}$ spectra of dextran- $\mathrm{NH}_{2}$ in $\mathrm{D}_{2} \mathrm{O}(\mathrm{a})$, MPEG-NHS in $\mathrm{CDCl}_{3}$ (b), and DexPEG block copolymer in $\mathrm{D}_{2} \mathrm{O}(\mathrm{c})$.

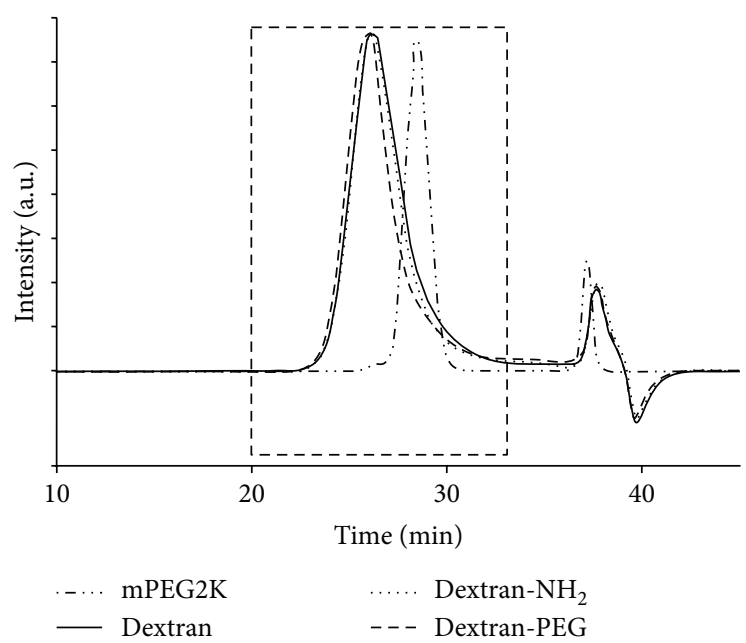

FIGURE 5: GPC chromatograms of DexPEG block copolymer.

of dextran. The molecular weight and conjugation yield were estimated from the proton NMR results. As shown in Figure 4, the sum of the peak intensity values of the protons at the 1 position of the glucose repeating unit can be assumed to be theoretically similar to those of both dextran- $\mathrm{NH}_{2}$ and the DexPEG block copolymer. Even though the other proton peaks of the glucose molecule of dextran and the ethylene proton of mPEG are difficult to separate, the ratio of the peak intensity of the protons at the 1 position of the glucose molecule to that of the protons at $3.3 \sim 4.2 \mathrm{ppm}$ changed before and after mPEG conjugation. From these changes of the proton peak intensities, the yield for the conjugation of mPEG to the end of dextran can be estimated. The calculated molecular weight and conjugation yield are summarized in Table 1. As shown in Table 1, the conjugation yield was about $84.8 \%$ and the M.W. was increased compared to that of dextran- $\mathrm{NH}_{2}$. As shown in Figure 5, GPC chromatograms showed the changes in the M.W.s of the polymers; that is, the DexPEG block copolymer revealed a decreased retention time compared to that of dextran and it showed single peaks. MPEG-NHS showed single peaks at $29 \mathrm{~min}$. Since no mPEG peaks were observed with the DexPEG peaks, it can be inferred that no unreacted $\mathrm{mPEG}$ remained in the conjugates. These results indicate that the DexPEG block copolymer was successfully synthesized by this procedure. Bosker et al. [17] reported that their attempts to couple long dextran (M.W. > $6,000 \mathrm{Da}$ ) were not successful. In spite of the fact that we used a longer dextran (weight average M.W. = 16,950 at Table 1), we were successful. Many trials have been reported to synthesize block copolymers composed of polysaccharide and PEG [14, $15,17,19-21]$. For example, block copolymer composed of mPEG and chitosan can be synthesized by free radical polymerization using potassium per sulfate as an initiator [19-21]. The yield of block copolymers was significantly controlled by 
initiator concentration and reaction temperature [20]. Furthermore, Kong et al. reported the self-assembly formation of mPEG-chitosan block copolymer [21]. Novoa-Carballal and Müller firstly synthesized mPEG-polysaccharide block copolymers by oxime click chemistry [22]. They successfully attached mPEG to the end of polysaccharide such as dextran, chitosan, and hyaluronic acid using oxime click reaction. However, oxime bond in the conjugates is labile at acidic $\mathrm{pH}$. Nanoparticles of mPEG-chitosan and/or lipophilic polymer were also reported for the delivery of anticancer drugs $[23,24]$. Previously, we reported that hyaluronic acid-PLGA (HAbLG) block copolymer was successively synthesized and self-assembled nanoparticles were fabricated for targeting of CD44 receptor of cancer cells [25].

In this report, we precisely synthesized/characterized block copolymer composed of dextran and $\mathrm{mPEG}$ by reductive amination of dextran with high yield. Since the hydroxyl functional group is abundant in the dextran domain, the DexPEG block copolymer will be a good candidate for use as a drug delivery vehicle.

\section{Conclusions}

DexPEG block copolymer was synthesized with aminated dextran and mPEG-NHS. The aminated dextran was prepared by reductive amination and showed the intrinsic peaks of both dextran at 3 5.5 ppm and hexamethylene diamine at $1 \sim 2.6 \mathrm{ppm}$ in the ${ }^{1} \mathrm{H}$ NMR analysis. MPEG-NHS was attached to the amine end group of the aminated dextran and the synthesized block copolymer was characterized using ${ }^{1} \mathrm{H}$ NMR and GPC. The M.W. and conjugation yield were estimated by comparing the intensity ratio of the proton peak of the glucose molecule (4.9 ppm and 3.3 4.0 ppm) to that of the ethylene group of PEG (3.7 ppm).

\section{Conflict of Interests}

The authors declare that they have no conflict of interests.

\section{Acknowledgment}

This study was supported by a grant from the Korean Healthcare technology R\&D Project, Ministry of Health \& Welfare, Republic of Korea (Project no. A091047).

\section{References}

[1] R. Terry, C. L. Yuile, A. Golodetz, C. E. Phillips, and R. R. White III, "Metabolism of dextran-A plasma volume expander. Studies of radioactive carbon-labeled dextran in dogs," The Journal of Laboratory and Clinical Medicine, vol. 42, no. 1, pp. 6-15, 1953.

[2] R. Mehvar, "Dextrans for targeted and sustained delivery of therapeutic and imaging agents," Journal of Controlled Release, vol. 69, no. 1, pp. 1-25, 2000.

[3] G. Wallenius, "Some procedures for dextran estimation in various body fluids," Acta Societatis Medicorum Upsaliensis, vol. 59, no. 1-2, pp. 69-77, 1953.

[4] L. Molteni, "Dextran and inulin conjugates as drug carriers," Methods in Enzymology, vol. 112, pp. 285-298, 1985.
[5] S. Sugahara, S. Okuno, T. Yano, H. Hamana, and K. Inoue, "Characteristics of tissue distribution of various polysaccharides as drug carriers: influences of molecular weight and anionic charge on tumor targeting," Biological and Pharmaceutical Bulletin, vol. 24, no. 5, pp. 535-543, 2001.

[6] K. Ichinose, N. Tomiyama, M. Nakashima et al., "Antitumor activity of dextran derivatives immobilizing platinum complex (II)," Anti-Cancer Drugs, vol. 11, no. 1, pp. 33-38, 2000.

[7] Y. Koyama, T. Miyagawa, A. Kawaide, and K. Kataoka, "Receptor-mediated absorption of high molecular weight dextrans from intestinal tract," Journal of Controlled Release, vol. 41, no. 3, pp. 171-176, 1996.

[8] Y. Pérez, A. Valdivia, H. L. Ramírez, and R. Villalonga, "Improved pharmacokinetics properties for catalase by sitespecific glycosidation with aminated dextran," Macromolecular Rapid Communications, vol. 26, no. 16, pp. 1304-1308, 2005.

[9] W. N. E. Van Dijk-Wolthuis, O. Franssen, H. Talsma, M. J. Van Steenbergen, J. J. Kettenes-van Den Bosch, and W. E. Hennink, "Synthesis, characterization, and polymerization of glycidyl methacrylate derivatized dextran," Macromolecules, vol. 28, no. 18, pp. 6317-6322, 1995.

[10] I.-S. Kim, Y.-I. Jeong, and S.-H. Kim, "Self-assembled hydrogel nanoparticles composed of dextran and poly(ethylene glycol) macromer," International Journal of Pharmaceutics, vol. 205, no. 1-2, pp. 109-116, 2000.

[11] I.-S. Kim, Y.-L. Jeong, D.-H. Kim, Y.-H. Lee, and S.-H. Kim, "Albumin release from biodegradable hydrogels composed of dextran and poly(ethylene glycol) macromer," Archives of Pharmacal Research, vol. 24, no. 1, pp. 69-73, 2001.

[12] D. Rutot, E. Duquesne, I. Ydens, P. Degée, and P. Dubois, "Aliphatic polyester-based biodegradable materials: new amphiphilic graft copolymers," Polymer Degradation and Stability, vol. 73, no. 3, pp. 561-566, 2001.

[13] Y.-I. Jeong, D. H. Kim, C.-W. Chung et al., "Doxorubicinincorporated polymeric micelles composed of dextran-bpoly(DL-lactide-co-glycolide) copolymer," International Journal of Nanomedicine, vol. 6, pp. 1415-1427, 2011.

[14] O. S. Hernandez, G. M. Soliman, and F. M. Winnik, "Synthesis, reactivity, and $\mathrm{pH}$-responsive assembly of new double hydrophilic block copolymers of carboxymethyldextran and poly(ethylene glycol)," Polymer, vol. 48, no. 4, pp. 921-930, 2007.

[15] G. M. Soliman and F. M. Winnik, "Enhancement of hydrophilic drug loading and release characteristics through micellization with new carboxymethyldextran-PEG block copolymers of tunable charge density," International Journal of Pharmaceutics, vol. 356, no. 1-2, pp. 248-258, 2008.

[16] G. Kwon, M. Naito, M. Yokoyama, T. Okano, Y. Sakurai, and K. Kataoka, "Micelles based on AB block copolymers of poly(ethylene oxide) and poly( $\beta$-benzyl L-aspartate)," Langmuir, vol. 9, no. 4, pp. 945-949, 1993.

[17] W. T. E. Bosker, K. Ágoston, M. A. Cohen Stuart, W. Norde, J. W. Timmermans, and T. M. Slaghek, "Synthesis and interfacial behavior of polystyrene-polysaccharide diblock copolymers," Macromolecules, vol. 36, no. 6, pp. 1982-1987, 2003.

[18] D.-G. Kim, Y.-I. Jeong, and J.-W. Nah, "All-trans retinoic acid release from polyion-complex micelles of methoxy poly(ethylene glycol) grafted chitosan," Journal of Applied Polymer Science, vol. 105, no. 6, pp. 3246-3254, 2007.

[19] F. Ganji and M. J. Abdekhodaie, "Synthesis and characterization of a new thermosensitive chitosan-PEG diblock copolymer," Carbohydrate Polymers, vol. 74, no. 3, pp. 435-441, 2008. 
[20] F. Ganji and M. J. Abdekhodaie, "The effects of reaction conditions on block copolymerization of chitosan and poly(ethylene glycol)," Carbohydrate Polymers, vol. 81, no. 4, pp. 799-804, 2010.

[21] X. Kong, X. Li, X. Wang et al., "Synthesis and characterization of a novel MPEG-chitosan diblock copolymer and self-assembly of nanoparticles," Carbohydrate Polymers, vol. 79, no. 1, pp. 170$175,2010$.

[22] R. Novoa-Carballal and A. H. E. Müller, "Synthesis of polysaccharide-b-PEG block copolymers by oxime click," Chemical Communications, vol. 48, no. 31, pp. 3781-3783, 2012.

[23] T.-W. Chung, D.-Z. Liu, J.-H. Hsieh, X.-C. Fan, J.-D. Yang, and J.-H. Chen, "Characterizing poly(e-caprolactone)-bchitooligosaccharide-b- poly(ethylene glycol) (PCP) copolymer micelles for doxorubicin (DOX) delivery: effects of crosslinked of amine groups," Journal of Nanoscience and Nanotechnology, vol. 6, no. 9-10, pp. 2902-2911, 2006.

[24] D. L. Tang, F. Song, C. Chen, X. L. Wang, and Y. Z. Wang, "A $\mathrm{pH}$-responsive chitosan-b-poly(p-dioxanone) nanocarrier: formation and efficient antitumor drug delivery," Nanotechnology, vol. 24, no. 14, Article ID 145101, 2013.

[25] Y.-I. Jeong, D. H. Kim, C.-W. Chung et al., "Self-assembled nanoparticles of hyaluronic acid/poly(dl-lactide-co-glycolide) block copolymer," Colloids and Surfaces B, vol. 90, no. 1, pp. 28$35,2012$. 

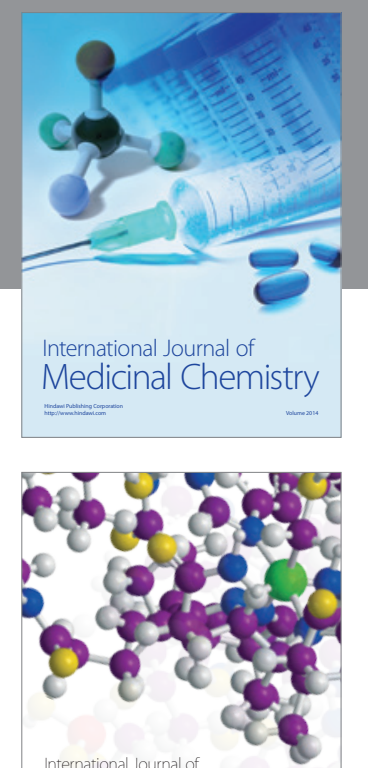

\section{Carbohydrate} Chemistry

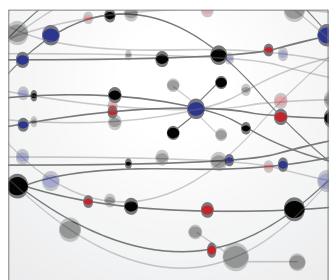

The Scientific World Journal
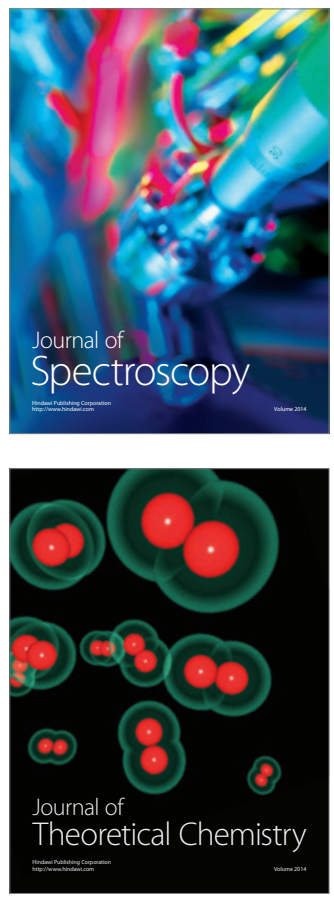
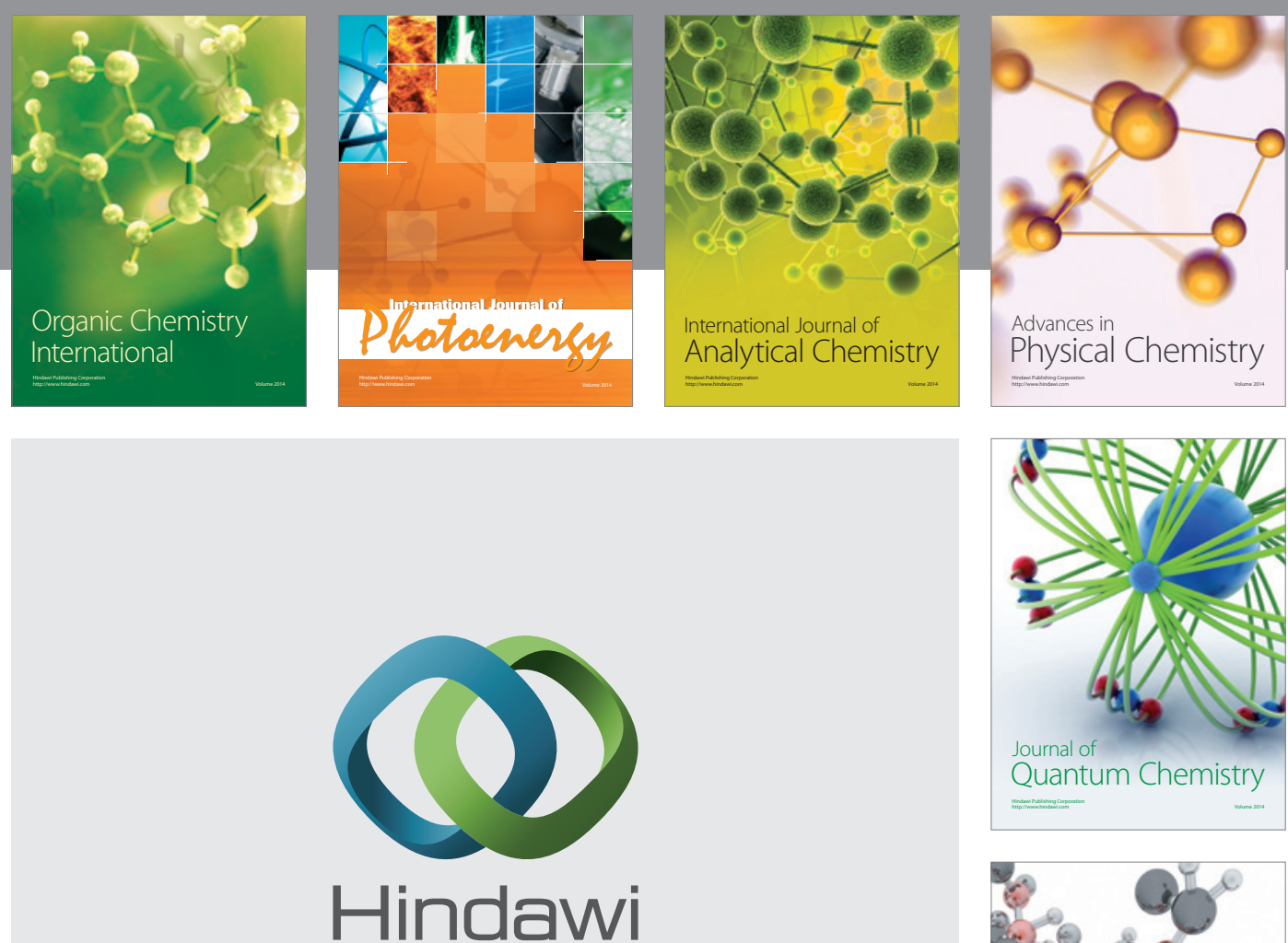

Submit your manuscripts at

http://www.hindawi.com

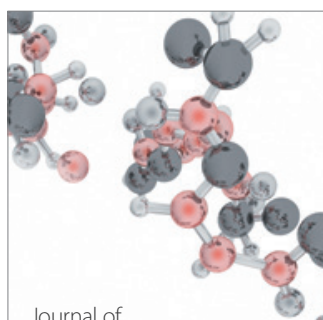

Analytical Methods

in Chemistry

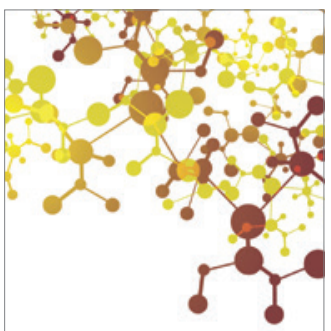

Journal of

Applied Chemistry

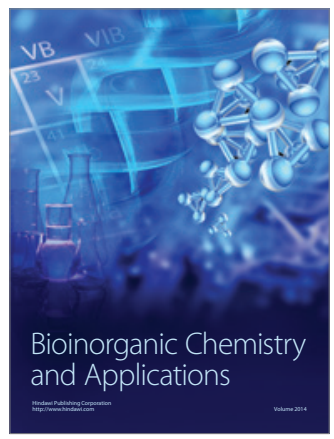

Inorganic Chemistry
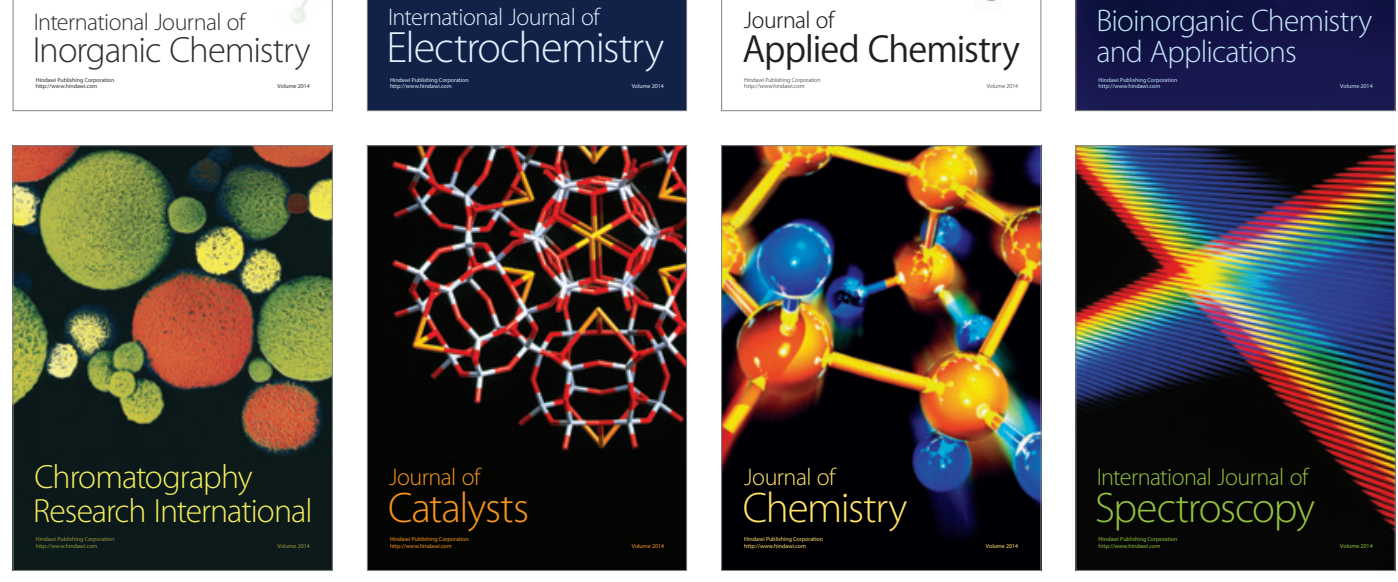